\title{
CONSULTA DE ENFERMAGEM: espaço para criação e utilização de protocolo para pacientes após revascularização miocárdica
}

F rancisca E lisângela T eixeira LIM A ${ }^{a}$, T helma L eite de ARAÚ J $0^{b}$, T hereza $M$ aria $M$ agal hães M OREIRAc, A lbeniza M esquita de M EDEIROSd, I res Lopes CU ST ÓDIOe, Elizabeth M esquita M ELO ${ }^{f}$

\section{RESUMO}

Estudo descritivo com objetivo de descrever um protocolo de consultas de enfermagem testado para pacientes após revascularização miocárdica. 0 protocolo, aplicado em uma unidade de cardiologia hospitalar, possui metas que devem ser atingidas no decorrer da consulta ou no período entre elas. Para o al cance de cada meta, foram estabel ecidas as ações de enfer magem que devem ser executadas pelo enfermeiro, expostas em três quadros: Estratégias de consulta de enfermagem referente ao primeiro atendimento; Estratégias de consultas de enfermagem referente aos atendimentos $n=2, n-3$ e $n-4$; E stratégias de consultas de enfermagem referente ao atendimento no 5 referente ao sexto mês após a cirurgia. A partir da aplicação do protocolo foi possível realizar uma avaliação sistemática do paciente, levantando seus reais problemas para planejar e realizar intervenções de enfermagem, principalmente as relacionadas às mudanças comportamentais.

D escritores: Processos de enfer magem. Continuidade da assistência ao paciente. Revascularização miocárdica.

\section{RESUMEN}

E studio descriptivo con objetivo de describir un protocolo de consultas de enfermería testado para pacientes después de la revascularización del miocardio. E I protocolo fue aplicado en unidad de cardiología hospitalaria, el cual posee metas que deben ser alcanzadas en el transcurso de la consulta o en el periodo entre ellas. Para el al cance de cada meta se establecieron las acciones de enfermería que deben ser ejecutadas por la enfer mera, expuestas en tres cuadros: Es strategias de consulta de enfermería referentes al primer atendimiento; E strategias de consultas de enfermería referentes a los atendimientos no 2, no 3 y $n-4$; E strategias referente al atendimiento no 5 referenteal sexto mes después de la cirugía. Se cree que la aplicación del protocolo posibilitará una evaluación sistemática del paciente, identificando sus problemas reales para la planificación y realización de inter venciones de enfermería, principalmente las relacionadas a los cambios de comportamiento.

Descriptores: P rocesos de enfermería. Continuidad de la atención al paciente Revascularización miocárdica.

T ítulo: Consulta deenfermería: espacio para la creación y utilización de protocol o para pacientes después dela revascularización del miocardio.

\section{ABST RACT}

A descriptive study aiming to describe a nursing consultation protocol tested for patients after myocardial revascularization. The protocol is implemented in a hospital cardiac unit, has goals that must be achieved during the consultation period or in between. F or reaching each goal, it has been established that nursing actions should be implemented by the nurse, displayed in three tables: Strategies for nursing consultation related to the first care; Strategies for nursing consultations related to the second, third and forth nursing visits; Strategies for nursing consultations related to the fifth nursing visit, referring to the sixth month after surgery. It is believed that the implementation of the protocol allows a systematic evaluation of the patient, raising their real problems, for planning and implementation of nursing interventions, especially those related to behavioral changes.

Descriptors: $\mathrm{N}$ ursing process. Continuity of patient care. M yocardial revascularization.

T itle: $\mathrm{N}$ ursing consultation: space for creation and use of protocol for patients after myocardial revascularization.

\footnotetext{
${ }^{\text {a } D ~ o u t o r a ~ e m ~ E ~ n f e r m a g e m, ~ P r o f e s s o r a ~ A ~ d j u n t a ~ d o ~ D ~ e p a r t a m e n t o ~ d e ~ E ~ n f e r m a g e m ~ d a ~ F ~ a c u l d a d e ~ d e ~ F ~ a r m a ́ c i a, ~ O ~ d o n t o l o g i a ~ e ~ E ~ n f e r ~ m a g e m ~}$ da U niversidade Federal do Ceará (DENF/ F F OE/ U FC), Fortaleza, Ceará, Brasil.

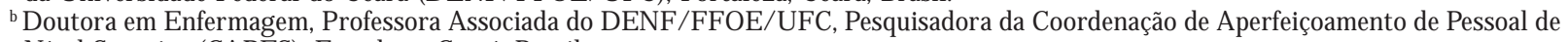
Nível Superior (CAPES), Fortaleza, Ceará, Brasil.

' D outora em Enfermagem, Professora da U niversidade Estadual do Ceará, Pesquisadora da CA PES, Fortaleza, Ceará, Brasil.

d Enfermeira E specialista em E ducação em Saúde Pública, Coordenadora do A mbulatório do H ospital de M essejana, Fortaleza, Ceará, Brasil.

e M estranda do Programa de Pós-Graduação em Enfermagem do DE N F F F OE / U FC, Enfermeira do Hospital de M essejana, Fortal eza, Ceará, Brasil.

f outora em Enfermagem, Professora da U niversidade de Fortaleza, Fortaleza, Ceará, Brasil.
} 


\section{INT RODUÇÃO}

As doenças crônicas cardiovasculares contribuem significativamente na mortalidade brasileira. São uma das principais causas de permanência hospitalar prolongada e de gastos públicos com hospitalizações e seu ônus econômico tem crescido exponencialmente nas últimas dé$\operatorname{cadas}^{(1,2)}$.

Assim, foi desenvolvido um estudo em Fortaleza, Ceará para averiguar a prática do autocuidado de pacientes após revascularização miocárdica (RM) quanto aos fatores de risco para a doença arterial coronariana (DAC), que constatou $50 \%$ de classificados entre bom e regular no grau de autocuidado, após um ano de RM ${ }^{(3)}$. A partir do estudo, buscou-se desenvolver estratégias para aumentar o autocuidado, controlando fatores de risco, minimizando complicações cardiovasculares pós-cirurgia e favorecendo melhoria da qualidade de vida dos pacientes.

D entre as estratégias de trabalho, escolheuse a consulta de enfermagem (CE), por ser um mé todo que possibilita a detecção precoce de desvios de saúde e acompanhamento de medidas instituídas, as quais se dirigem ao bem-estar dos envolvidos. É uma ação que se diferencia entre as várias maneiras de cuidar, pois possibilita aproximação pessoa a pessoa, estabelecendo uma relação interpessoal de ajuda concreta diante das variáveis culturais $^{(4)}$.

A consulta de enfermagem foi criada há mais de quarenta anos, contudo poucos estudos têm sido desenvolvidos para avaliar a eficácia da sua implementação. No intuito de contribuir para sanar essa lacuna, este estudo visa o desenvolvimento e experimentação de um Protocolo de Consultas de Enfermagem (PCE) para o paciente submetido à cirurgia de RM .

A utilização do protocolo ao pós revascularizado miocárdico justifica-se pela sua necessidade de passar por uma reabilitação dinâmica, orientada à saúde e capaz de auxiliá-lo a atingir o maior nível possível de funcionamento físico, mental, espiritual e econômico. Tal processo o ajuda a alcançar qualidade de vida com dignidade, auto-estima e independência ${ }^{(5)}$.

Diante dessas consider ações, tem-se como objetivo descrever um Protocolo de Consultas de E nfermagem testado para pacientes após RM .

\section{METODOLOGIA}

0 estudo descreve um protocolo aplicado em unidade ambulatorial de cardiologia de Fortaleza, Ceará, conveniado ao Sistema Ú nico de Saúde (SU S). A escolha do local se deu por se tratar de serviço de grande abrangência na atenção à saúde da população, pois atende pacientes cardiopatas de todo o Ceará e de outros estados das regiões $\mathrm{N}$ ordeste e N orte.

O PCE foi testado com adultos e idosos de ambos os sexos com RM, pelo desenvolvimento de um ensaio clínico randomizado avaliador da eficácia do protocolo ${ }^{(6)}$.

Foram critérios de inclusão: ter realizado $R M$, independente do tipo e do número de pontes (safena ou mamária); idade superior a 18 anos; estar consciente, deambulando independente; e ser capaz de se locomover até 0 ambulatório de real ização da CE com utilização do protocolo. Além disso, deviam ter acompanhamento médico, conforme rotina institucional. Foram critérios de exclusão: residentes de fora da região metropolitana de Fortaleza, pois seu deslocamento seria muito oneroso.

A tenderam aos critérios de inclusão 82 pacientes, dos quais 42 pertenciam ao grupo controle (GC) e 40 ao grupo de intervenção (GI). E m cada grupo foi descontinuado um paciente, por óbito. E no grupo controle, dois pacientes não retornaram para a revisão pós-operatória ambulatorial, mesmo após diversos contatos telefônicos, sendo descontinuado do estudo. Então cada grupo ficou constituído por 39 pacientes.

Para realizar o ensaio clínico randomizado foi criado o grupo controle e o grupo de intervenção, cuja randomização ocorreu da forma descrita a seguir.

Grupo controle: realizaram cirurgia de RM nos dias ímpares $(1,3,5,7 \ldots 31)$. Estes pacientes foram acompanhados no pós-operatório após a alta hospitalar conforme o método convencional de tratamento - somente pelo cardiologista no ambulatório, acrescido do acompanhamento, em dois momentos, pela pesquisadora. A avaliação dos pacientes do grupo controle pela pesquisadora ocorreu primeiro enquanto estavam internados na enfermaria, no dia ou na véspera da alta e, em um segundo momento, seis meses após a alta hospitalar, coincidindo com a consulta médica. 
G rupo de intervenção: passaram por cirurgia de RM nos dias pares $(2,4,6 \ldots 30)$. Foram submetidos ao programa de intervenção de enfermagem, após a primeira avaliação da pesquisadora realizada no dia ou na véspera da alta hospitalar. As consultas subsequentes foram implementadas a cada um, dois, quatro e seis meses. A pós alta hospitalar os pacientes do GI eram acompanhados pelos médicos da instituição igual aos do GC.

O PCE foi elaborado para seguimento pelo período de seis meses, contendo metas a serem atingidas no decorrer da consulta ou no período entre elas. Para o alcance de cada meta foram estabelecidas ações de enfer magem a serem exe- cutadas pelo enfermeiro, sendo que algumas metas e ações se repetem em virtude do acompanhamento da evolução do paciente.

0 estudo foi aprovado pelo Comitê de Ética da mencionada instituição sob parecer no 292/ 05. Todos os participantes dos dois grupos anuíram com a pesquisa e assinaram Termo de Consentimento Livre e E sclarecido.

\section{RESULT AD OS}

Como resultados, temos a descrição do PCE para pacientes submetidos à RM, segundo 0 atendimento realizado, conforme exposto nos Quadros 1,2 e 3.

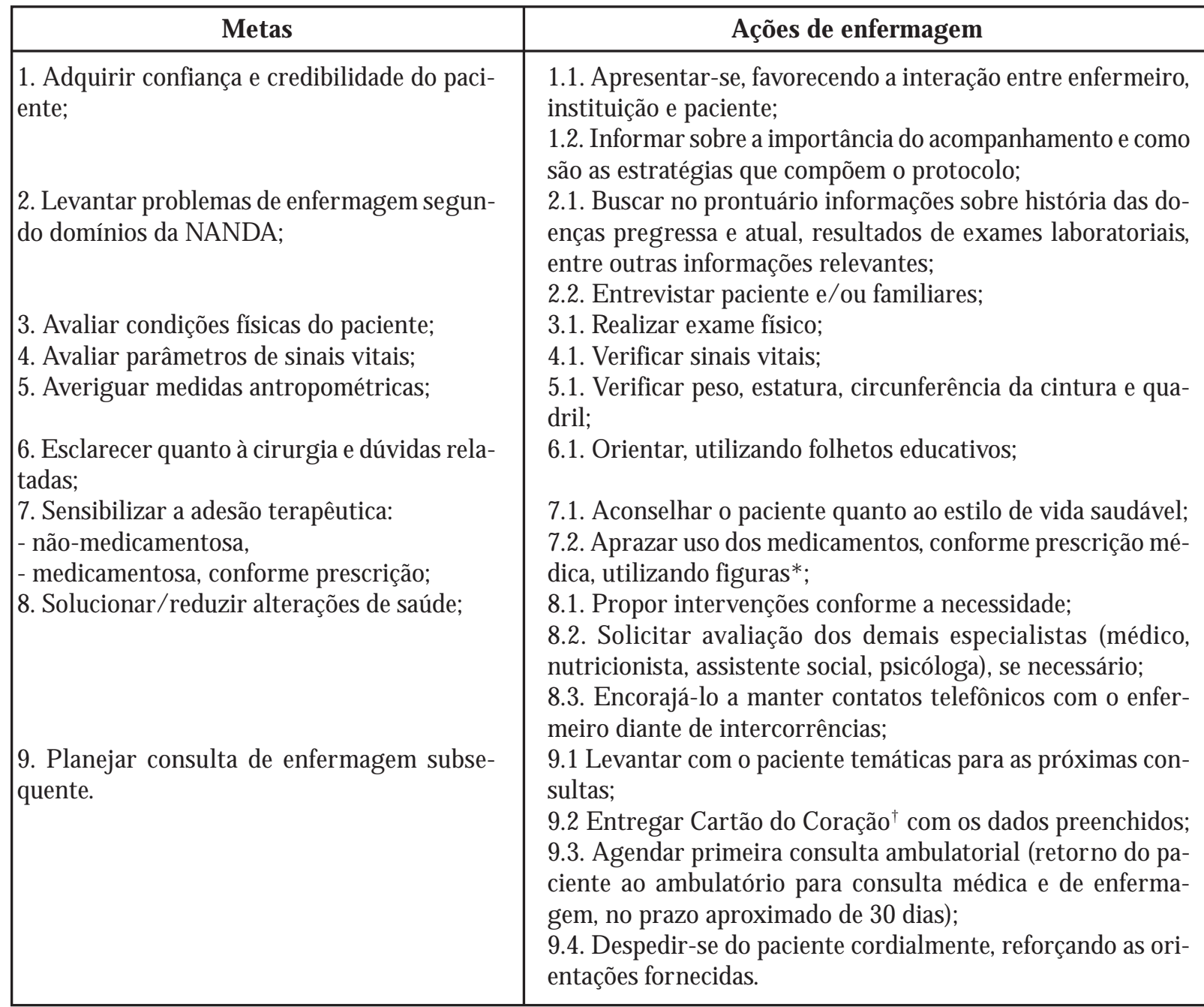

Quadro 1 - Estratégias de consulta de enfermagem referente ao primeiro atendimento, na unidade de inter namento. Fortaleza, CE, 2007. * U m quadro contendo cinco colunas: 1 - medicamento, 2 - dose, 3 - manhã (figura do sol nascendo), 4 - tarde (figura do sol inteiro), 5 - noite (figura da lua). 0 preenchimento deve ser um desenho de um comprimido com quatro quadrantes e pintar com lápis de cor a dose prescrita, nos horários correspondentes à prescrição médica.

${ }^{\dagger}$ No cartão do coração, consta dados pessoais do paciente (no prontuário, nome, endereço, tel efone, data da cirurgia), dados da doença arterial coronariana e cirurgia de revascularização miocárdica (definição, fatores de risco e manifestações clínicas), prática de autocuidado após a alta hospitalar (mudanças no estilo de vida), e dados clínicos (sinais vitais, peso, índice de massa corporal e glicemia) com espaço para registro dos dados durante a consulta de enfermagem. 


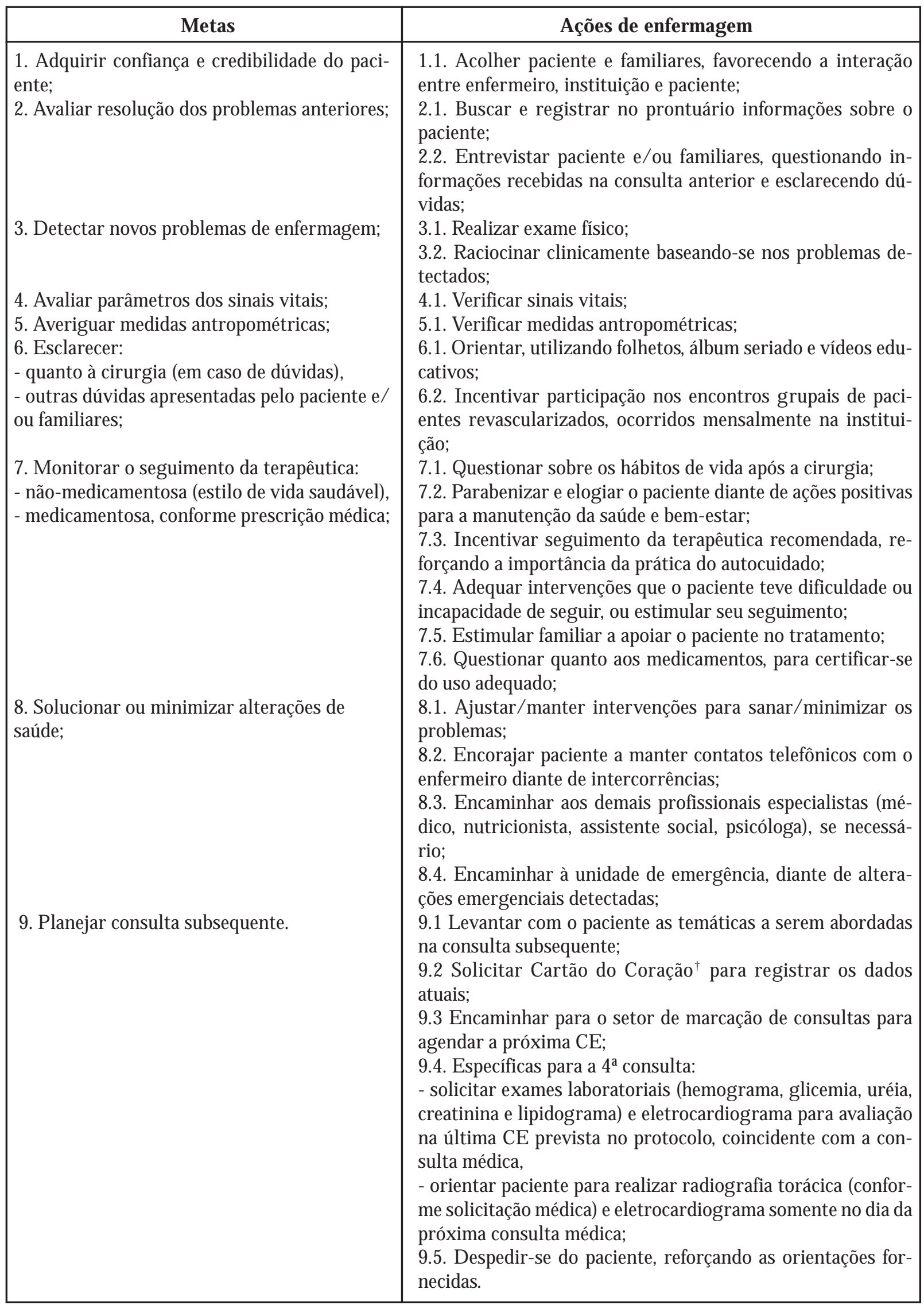

Q uadro 2 - Estratégias de consultas de enfermagem referente aos atendimentos № 2, № 3 e nํ4, no ambulatório. Fortaleza, CE, 2007. 


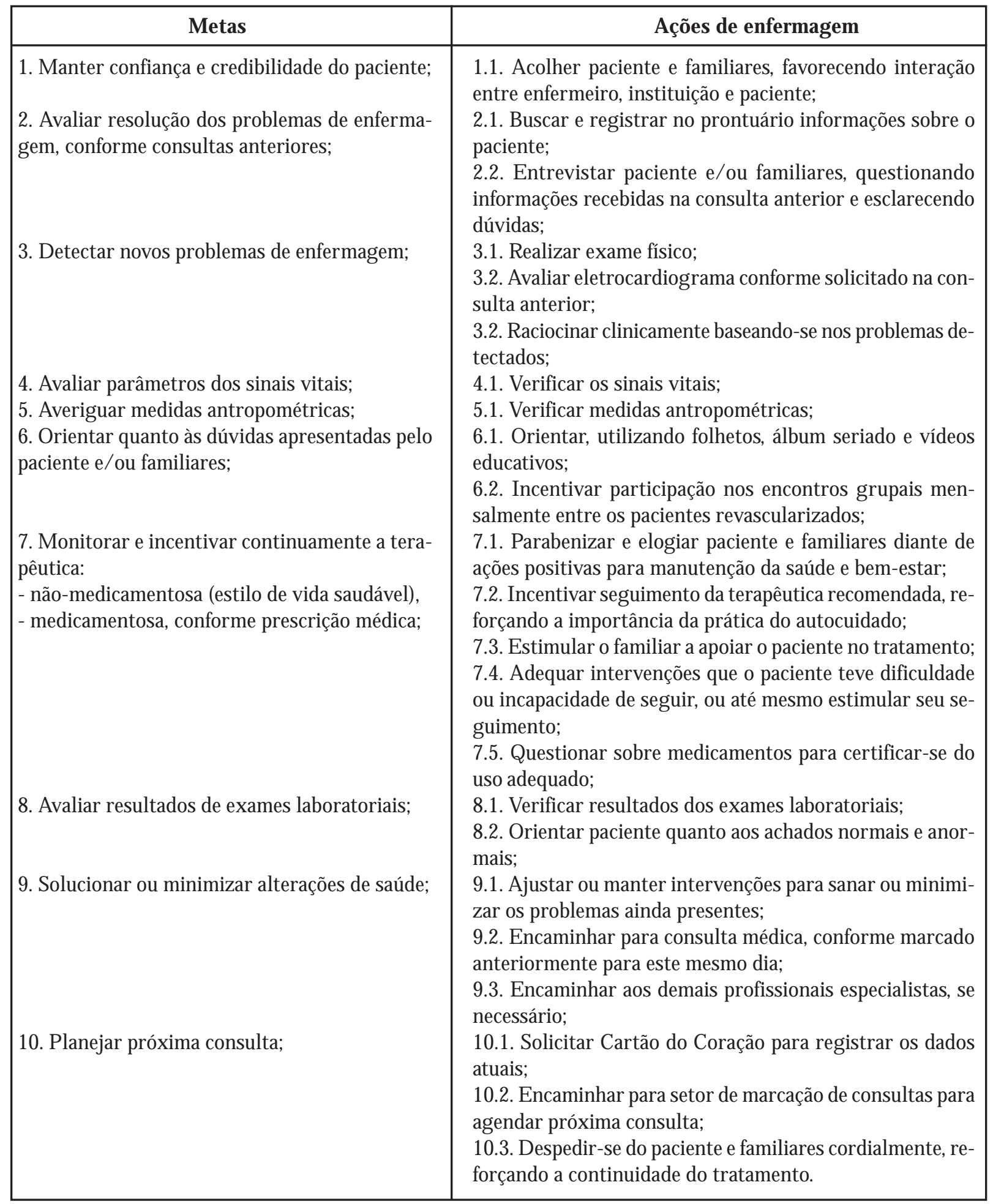

Q uadro 3 - Estratégias de consultas de enfermagem referente ao atendimento № 5 no ambulatório, relativo ao sexto mês após a cirurgia. Fortaleza, CE, 2007.

Para melhor visualização do PCE, elaborouse um diagrama, com os seguintes elementos: fatores de risco da DAC modificáveis e comportamentos individuais de minimização ou, pelo menos, de controle desses fatores (F igura 1 ).
E ste diagrama foi construído a partir da identificação dos fatores de risco modificáveis da DAC e dos comportamentos humanos favoráveis para controlar, minimizar ou prevenir o surgimento destes fatores. 


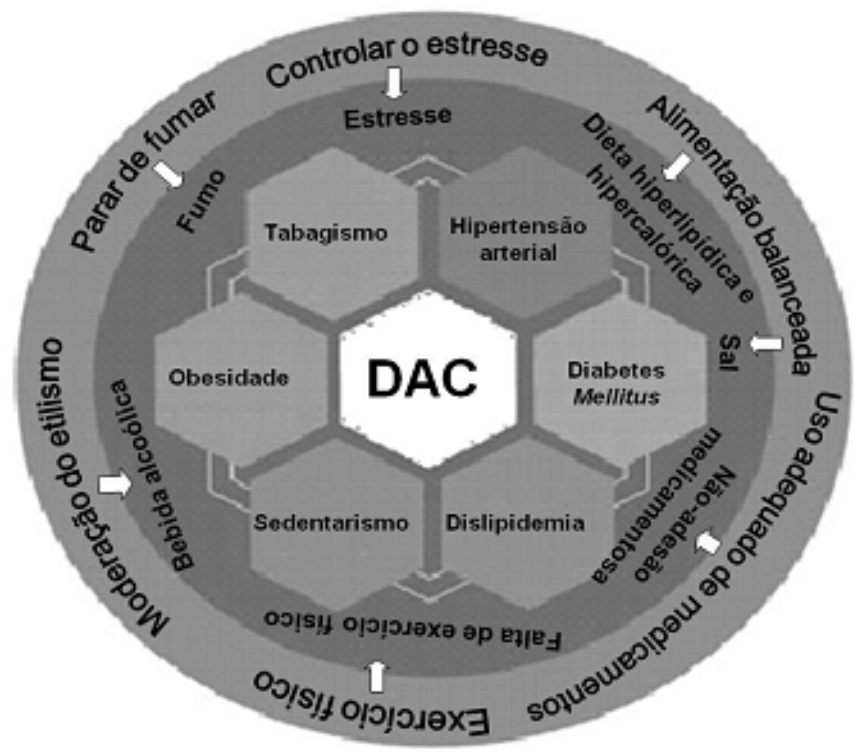

Figura 1 - Diagrama de condutas comportamentais para controle dos fatores de risco modificáveis e controláveis da doença arterial coronariana (DAC).

0 diagrama da DAC deve ser analisado do interior para o exterior. No interior estão apresentados os fatores de risco para DAC: hipertensão arterial, diabetes mellitus, dislipidemia, obesidade, sedentarismo e tabagismo. Estes fatores são comportamentais e estão inter-relacionados. São decorrentes de hábitos de vida inadequados, como dieta hiperlipídica, hipersódica e hipercalórica, falta de exercício físico, estresse, fumo, bebidas al coólicas, uso incorreto ou mesmo não utilização dos medicamentos, dentre outros. T ais fatores podem ser minimizados ou evitados com mudanças comportamentais, listadas na linha mais externa do diagrama, como: alimentação balanceada, controle do estresse, abstinência do tabagismo e etilismo, exercício físico e uso de medicamentos conforme prescrição médica.

\section{Recursos e técnicas utilizadas na operacionalização do PCE}

D urante a CE, o enfermeiro-pesquisador enfatizava promoção, proteção e recuperação da saúde, tendo o paciente como sujeito de suas ações. Porém, simultaneamente, enfermeiro e paciente buscavam identificar os problemas e priorizar sua resolubilidade.

Para o seguimento do protocolo foram estabelecidos recursos e técnicas de avaliação dos pacientes na consulta de enfermagem. Para tanto, 0 protocolo inicia-se com o levantamento de pro- blemas, por ser o início do relacionamento enfermeiro-paciente. É a fase de coleta de informações sobre a situação de saúde, na busca de evidências de funcionamento anor mal ou fatores de risco que contribuam para problemas de saúde. Com esta finalidade, 0 instrumento contemplou os 13 domínios de enfer magem propostos pela $\mathrm{N}$ orth A merican $\mathrm{N}$ ursing Diagnosis A ssociation (NANDA) ${ }^{(7)}$, além do exame físico.

Para o levantamento dos problemas foi utilizada a entrevista, por possibilitar a coleta de dados precisos e completos sobre o estado de saúde da pessoa ${ }^{(8)}$. Ela foi realizada em ambiente apropriado, tranquilo e confidencial, espontaneamente. Quanto ao exame físico, para sua realização foram utilizados os métodos propedêuticos, quais sejam: inspeção, pal pação, percussão e ausculta, no sentido céfalo-caudal, al ém da verificação dos sinais vitais ${ }^{(8,9)}$.

Dos sinais vitais, salientou-se a pressão arterial (PA), por se tratar de um indicador de avaliação deste estudo. Para medi-la foi utilizado um esfigmomanômetro de coluna de mercúrio, variando de 0 a $300 \mathrm{mmH} \mathrm{g}$, e estetoscópio. A técnica adotada seguiu as recomendações da A merican $\mathrm{H}$ eart Association ${ }^{(10)}$.

No momento inicial do estudo, considerou-se o paciente com hipertensão arterial aquele que tinha diagnóstico médico estabelecido. $E$ nas avaliações posteriores, foi utilizado como indicador de pressão arterial não controlada o que apresentasse $P A \geq 140 \times 90 \mathrm{mmH} \mathrm{g}^{(10)}$. 
Os indicadores de avaliação dos dados antropométricos seguiram os parâmetros recomendados pela literatura ${ }^{(8,9)}$, sendo verificados peso e estatura para análise do índice de massa corporal (IMC), circunferência da cintura (CC) e relação cintura e quadril (RCQ).

Para verificação de peso e estatura, utilizouse balança antropométrica digital com variação de 1 a 150 kg, graduado de 100 em 100 g. A estatura foi mensurada com a régua antropométrica, existente na referida balança, medindo até $2 \mathrm{~m}$ com graduação de $0,5 \mathrm{~cm}$. Para mensuração dessas medidas, o paciente foi orientado a subir na balança descalço, mantendo-se de pé, com pés juntos e voltados para frente, ombros relaxados e braços ao longo do corpo.

0 indicador mais usado para avaliação do peso em relação à altura foi proposto por Quetelet, e é conhecido por índice de massa corporal (IM C), calculado pela fórmula: peso $(\mathrm{em} \mathrm{kg}$ ) dividido pelo quadrado da altura (em metros). Os valores de referência são: baixo peso (IM C $\left.<20 \mathrm{~kg} / \mathrm{m}^{2}\right)$, normal (I M C de 20 a 24,99 kg/ m²), sobrepeso (I M C de 25 a 29,99 kg/ m²), obesidade (I M C de 30 a 39,99 $\left.\mathrm{kg} / \mathrm{m}^{2}\right)$, obesidade grave $\left(\mathrm{IMC} \geq 40 \mathrm{~kg} / \mathrm{m}^{2}\right)^{(8,9)}$.

Verificou-se, ainda, a CC, que foi medida com fita métrica inelástica, numerada até $150 \mathrm{~cm}$, com definição de medida de $0,1 \mathrm{~cm}$. 0 participante ficava na posição ortostática, com o mínimo de roupa possível, e a fita era colocada na distância média entre a última costela flutuante e a crista ilía$\mathrm{ca}^{(8)}$. Segundo a Organização M undial de Saúde (OM S), os riscos de complicações metabólicas estão aumentados quando a CC é superior a $94 \mathrm{~cm}$ em homens e $80 \mathrm{~cm}$ em mulheres e muito aumentados quando superior a $102 \mathrm{~cm}$ e $88 \mathrm{~cm}$, respectivamente ${ }^{(11)}$. Porém, há autores que consideram a CC normal em homens até $102 \mathrm{~cm}$ e em mulheres até $88 \mathrm{~cm}^{(9)}$.

Para a constatação do tipo de obesidade andróide ou ginecóide, utilizou-se a relação cintura/ quadril, obtida pela divisão da circunferência da cintura abdominal pela circunferência do quadril do paciente. A verificação da CC, num ponto médio entre o final dos arcos costais, e a do quadril (Q) ao nível das cristas ilíacas. Os valores de referência de normalidade são os seguintes: homens $(R C Q<0,9)$ e mulheres (RCQ $<0,8)$. É aceito que existe risco metabólico quando a relação cinturaquadril é maior do que 0,9 no homem e 0,8 na muIher $^{(9)}$.
Quanto aos exames bioquímicos, foram realizados na própria instituição e o sangue foi colhido pelos técnicos de laboratório. Todos foram orientados ao jejum por doze horas antes da coleta do sangue. Foram realizadas dosagens de colesterol total, triglicerídeos e glicemia.

Foi considerada dislipidemia a hipercolesterolemia isolada (aumento do colesterol total e/ ou da fração LDL-colesterol), hipertrigliceridemia isolada (aumento dos triglicerídeos), hiperlipidemia mista (aumento do colesterol total e triglicerídeos) e diminuição isolada do HDL-colesterol ou associada a aumento de triglicerídeos ou LDL-colesterol. Foi referência para diagnóstico de dislipidemias: colesterol total $\geq 200 \mathrm{mg} / \mathrm{dl}$; LD L colesterol $\geq 160 \mathrm{mg} / \mathrm{dl}$; HDL-colesterol < 40mg/ $\mathrm{dL}$; triglicerídeos $\geq 150 \mathrm{mg} / \mathrm{dl}^{(12,13)}$.

A glicemia foi realizada em jejum - sem ingestão calórica por, no mínimo, 8 horas. Foi considerado glicemia nor mal $<100 \mathrm{mg} / \mathrm{dl}$, tolerância a glicose diminuída $>100 \mathrm{a}<126 \mathrm{mg} / \mathrm{dl}$, e diabetes mellitus $\geq 126 \mathrm{mg} / \mathrm{dl}^{(14)}$.

Para ser bem-sucedido, o tratamento medicamento deve seguir as recomendações das $D$ iretrizes de Cirurgia de Revascularização M iocárdica(15), as quais são: ácido acetil salicílico (AAS) (100-325mg/ dia), iniciando nas primeiras $24 \mathrm{~h}$ de pós-operatório e mantido indefinidamente, e em caso de intolerância ao A AS, é indicado ticlopidina $250 \mathrm{mg} / \mathrm{dia}$; inibidores da enzima conversora de angiotensina (ECA), em todos os pacientes com DAC, diabéticos ou não, e/ ou que apresentem disfunção sistólica ventricular; bloqueadores dos canais de cálcio, de segunda geração, por período de seis meses pós implante da artéria radial; e estatina infinitamente em dislipêmicos. A s orientações medicamentosas foram realizadas de acordo com as prescrições médicas. Contudo, as não-medicamentosas seguiram tanto as últimas recomendações das D iretrizes(10), quanto as de diver sos estudiosos sobre doenças cardiovasculares $(1,5,13,15,16)$.

0 tratamento deve ser implementado em todos os pacientes. Cada um deve receber antiplaquetário e estatina, independentemente do nível de colesterol (17).

Quanto ao estilo de vida, devem-se seguir as recomendações: 1) manter o peso corporal na faixa normal do I M C ; 2) padrão alimentar saudável (consumir: alimentos com baixa densidade calórica; dieta rica em frutas e vegetais; dieta hipolipídica, hipoglicídica (se for diabético); comidas lights 
e fibras; baixo teor de gorduras saturadas e totais); 3) reduzir a ingestão de sódio para não mais de $6 \mathrm{~g}$ sal $/$ dia (4 colheres de café rasas de sal $=4 \mathrm{~g}+2 \mathrm{~g}$ de sal próprio dos alimentos); dieta hipossódica, no caso de hipertensão ou disfunção de ventrículo esquerdo; 4) habituar-se ao exercício físico regular (Ex.: caminhadas contínuas); enfatizar o aumento progressivo dos exercícios, iniciando por 10 minutos diários e aumentar cinco minutos por semana até pelo menos $30 \mathrm{~min} / \mathrm{dia}$, 3-5 vezes/ semana; Prescrever hidroginástica, ioga ou relaxamento de acordo com a capacidade funcional; 5) abstinência do etilismo (embora seja recomendado reduzir consumo a 30g/ dia (homens), 15g/ dia (mulheres), optou-se por aconselhar 0 abandono do etilismo); 6) abstinência do cigarro (observar o controle do peso); 7) controlar o estresse emocional (abordar aspectos psicossociais e emocionais relacionados à cirurgia e controle pós-operatório, pois ajuda na adesão à terapêutica) ${ }^{(10,15)}$.

\section{CONCLUSÕES}

A CE ao paciente submetido à RM foi fundamental. Contudo, acredita-se que a obtenção de resultados satisfatórios só foi possível em virtude da utilização do PCE, o qual possibilitou uma avaliação sistemática do paciente, pois foram levantadas seus reais problemas para o planejamento e realização de intervenções de enfermagem, estabelecendo prioridades na adoção de medidas, principalmente, das que envolvam mudanças de comportamento, hábitos e estilo de vida.

0 estudo foi aplicado em unidade ambulatorial. Contudo, desenvolvido para aplicação em qualquer ambiente adequado, tais como: ambulatório de cardiologia, público ou privado; unidade de saúde com atendimento ao paciente após RM .

O PCE foi testado com pacientes adultos e idosos de ambos os sexos que realizaram RM, sendo constatada sua eficácia nas mudanças comportamentais do estilo de vida dos pacientes, tais como melhoria na qualidade da dieta; adesão à prática de exercício físico; e redução/ abstinência do tabagismo e de bebidas alcoólicas. T ais mudanças comportamentais foram positivas para redução de fatores de risco e, consequentemente, para minimização de complicações cardiovasculares.

Contudo, recomenda-se que as avaliações sejam periódicas e sucessivas, e permitam comparação precisa no progresso do paciente, determinan- do se houve evolução positiva ou negativa. D essa forma, é possível modificar o protocolo, adequando-o à realidade do paciente.

E ssa abordagem individualizada, pela consulta de enfermagem, para o cuidado com o paciente, requer dos enfermeiros o emprego de seu tempo, conhecimento, competência e planejamento para melhorar a qualidade da assistência e obter resultados satisfatórios na manutenção ou recuper ação da saúde do paciente.

\section{REFERÊNCIAS}

1 Castro LCV, F ranceschini SCC, Priore SE, Pelúzio M CG. N utrition and cardiovascular diseases: the risk markers in adults. Rev N utr. 2004;17(3):369-77.

2 D'A lencar ER, Lima M M R, M endonça PM L, Custodio IL, D'Alencar BP, L ima FET. A çõ̃es de educação em saúde no controle do sobrepeso/ obesidade no ambiente de trabal ho. Rev Rene. 2010;11(1):172-80.

3 Lima FET. A prática do autocuidado após revascularização do miocárdio [ dissertação] . Fortaleza: D epartamento de E nfermagem, U niver sidade F ederal do Ceará; 2002.

4 Zagonel IPS. Consulta de enfermagem: um modelo de metodologia para o cuidado. In: W estphal en MEA, Carraro T E, organizadores. M etodologias para a assistência de enfermagem: teorizações, model os e subsídios para a prática. G oiânia: AB; 2001. p. 41-56.

5 D oença crônica. In: Smeltzer SC, Bare BG. Brunner \& Suddarth: tratado de enfermagem médico-cirúrgica. 10ª ed. Rio de J aneiro: Guanabara Koogan; 2006. p. 153-64.

$6 \mathrm{LimaFET}$. Protocolo de consultas de enfermagem ao paciente após a revascul arização do miocárdio: avaliação da eficácia [ dissertação] . Fortaleza: D epartamento deE nfer magem, U niversidade F ederal do Ceará; 2007.

7 N orth A merican N ursing Diagnosis Association. Diagnósticos de enfer magem da NAN DA : definições e classificação 2009-2011. Porto A legre: Artmed; 2010.

8 Jarvis C. Exame físico e avaliação em saúde. Rio de Janeiro: G uanabara K oogan; 2002.

9 Porto CC. Semiologia médica. 5a ed. Rio de Janeiro: Guanabara Koogan; 2005.

10 VI Diretrizes Brasileiras de Hipertensão Arterial. Rev Bras Hipertens. 2010;17(1):7-60. 
11 W orld $\mathrm{H}$ ealth O rganization. O besity: preventing and managing the global epidemic. G eneva; 1998.

12 W orld H ealth Organization. Physical status: the use and inter pretation of anthropometry. G eneva; 1995.

13 III Diretrizes Brasileiras sobre D islipidemias e Diretriz de Prevenção da A terosclerose do Departamento de Aterosclerose da Sociedade Brasileira de Cardiologia. Arq Bras Cardiol. 2001;77(Supl 3):1-48.

14 Sociedade Brasileira de Diabetes. T ratamento e acompanhamento do diabetes mellitus: diretrizes da Sociedade Brasileira de Diabetes. São Paulo; 2008.
15 Diretrizes da cirurgia de revascularização miocárdica, val vopatias e doenças da aorta. A rq B ras Cardiol. 2004;82(Supl 5):1-20.

16 Y usuf S, Hawken S, O unpuu S, D ans T, Avezum A, $L$ anas $F$, et al. E ffect of potentially modifiable risk factors associated with myocardial infarction in 52 countries (the INTERHEART study): case-control study. L ancet. 2004;364(9438):937-52.

17 Florenzano UF. Opciones terapéuticas en enfermedad coronaria. Rev M éd Clín Condes. 2009;20 (3):336-9.

\section{Endereço da autora / Dirección del autor / Author's address:}

Francisca E lisângela T eixeira Lima

Av. dos Expedicionários, 3406, ap. 1203, BI. 1, Benfica 60410-410, Fortaleza, CE

E-mail:felisangela@yahoo.com.br
Recebido em: 24/ 05/ 2010

A provado em: 16/ 08/ 2010 